\title{
A WORLD ARRAY VLBI IMAGE OF 3C216 AT 1.6GHZ
}

\author{
C.E. AKUJOR AND R.W. PORCAS \\ Max-Planck-Intitut fuer Radioastronomie, Bonn, Germany \\ AND
}

I. FEJES

FOMI Satellite Geodetic Observatory, Budapest, Hungary

The quasar $3 \mathrm{C} 216$ has the distinction of being classified as a blazar as well as a compact steep-spectrum radio source (Akujor et al, 1993). It has a grossly mis-aligned large-scale radio structure. Previous VLBI images include those of the core region (Barthel et al 1988; Venturi et al 1993) with 1 mas resolution, and the sub-kpc scale core-jet with resolution ca. 25 mas (Fejes et al, 1992; Akujor et al, 1993). In order to investigate the connection between these structures seen on different scales, we have made a $1.6 \mathrm{GHz}$ observation of the core-jet region with a "World Array" VLBI network. This consisted of 20 antennas, at Effelsberg (Germany), Jodrell "Lovell" (UK), Westerbork (Netherlands), Medicina and Noto (Italy), Onsala-26m (Sweden), Torun-15m (Poland), Simeiz (Ukraine), Bear Lakes (Russia), Seshan (China), Hartebeesthoek (S.Africa), Green Bank-43m, VLA and the VLBA antennas at HN, NL, PT, KP, LA, BR, OV (USA). The observations were made on 18 November, 1992, using the Mk2 recording system, and these were amongst the last to be correlated using the JPL-Caltech Block2 processor. The NRAO AIPS package was used for the data analysis.

Images of 3C216 made with both uniform and natural weighting of the uv data are shown in Fig 1a,b. They show a core, and jet emission following a gently curving path, with underlying wiggles, ending in a sharp bend ca. $1 \mathrm{kpc}$ from the core. Beyond this point the jet gradually fades in intensity through a string of weak components. The fairly bright knot at the bend appears to be edge-brightened; this, and the flatter spectrum of the knot (Akujor et al, 1993) may support the idea that the jet is being deflected by a gas cloud or clump. The radio emission from the jet fades about half way 

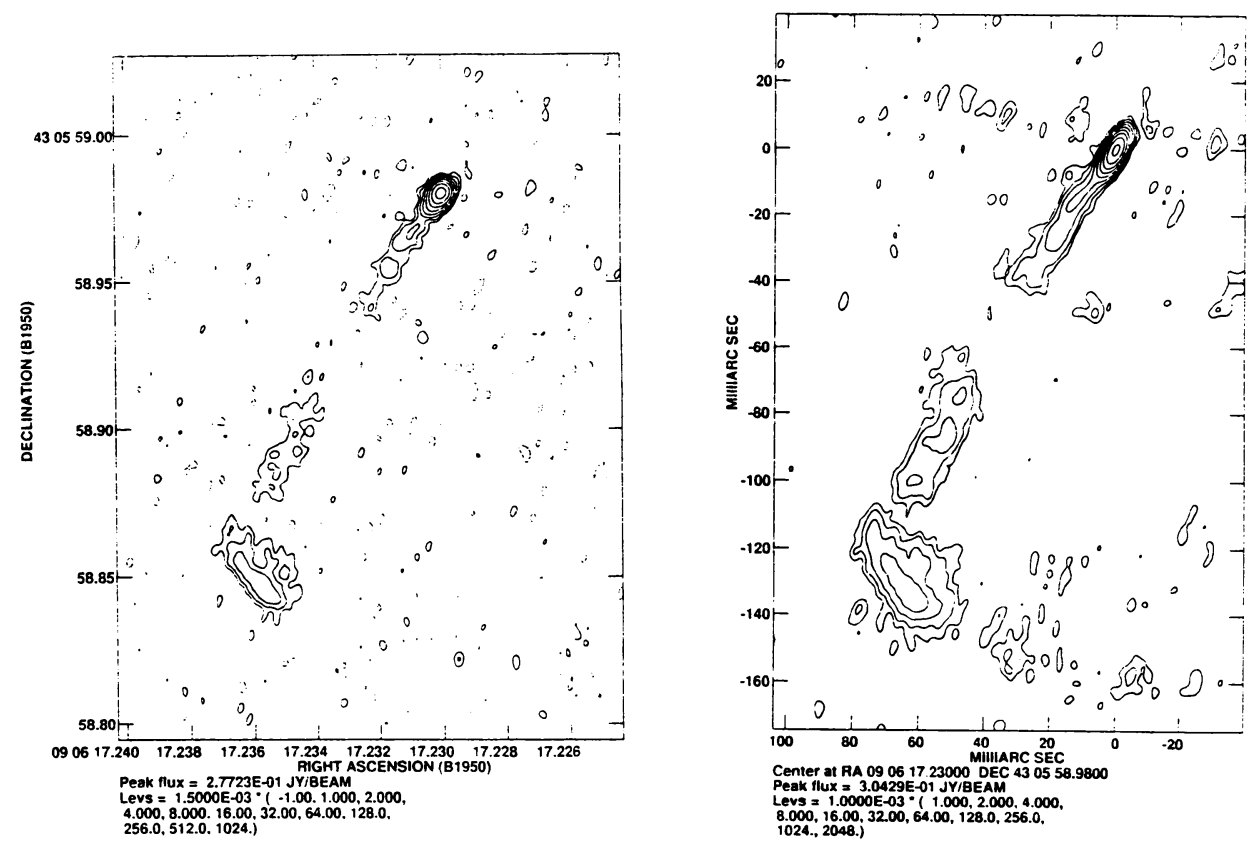

Figure 1. World array images of $3 \mathrm{C216}$ at $1.6 \mathrm{GHz}$ : (a, left) Uniform weighting, beam $4.5 \times 4.5$ mas; (b, right) Natural weighting, beam $6.7 \times 3.5$ mas, pa -24 .

between the core and this knot, but reappears well before the bend. This feature is prominent in the 0.61 GHz VLBI map of Fejes et al (1992).

\section{References}

Akujor, C.E. et al, 1993, in Subarcsecond Radio Astronomie, Ed Davies, R.J. and Booth, R.S., CUP, p265

Barthel, P.D. et al, 1988, ApJ, 329, L51

Fejes, I., Porcas, R.W. and Akujor, C.E., 1992, $A \& A, 257,459$

Venturi, T. et al, 1993, $A \& A, 271,65$ 